\title{
Primary patency rates of endovascular interventions in hemodialysis patients with central venous stenosis and occlusions
}

\author{
Mehmet Burak Çildağ, Veli Süha Öztürk, Recep Özgür, Ömer Faruk Kutsi Köseoğlu \\ Department of Radiology, Faculty of Medicine, Adnan Menderes University, Aydin, Turkey
}

\begin{abstract}
Introduction: The purpose of this study was to determine the primary patency rate of endovascular interventions in hemodialysis patients who had central venous stenosis or occlusion.

Material and methods: Twenty-seven hemodialysis patients, who underwent endovascular intervention between January 2013 and January 2018 for central venous stenosis or total obstruction, were included in the study. Endovascular interventions consisted of percutaneous transluminal angioplasty (PTA) or stent implantation. Primary patency rate of endovascular intervention at the sixth and twelfth months were evaluated.

Results: Stent implantation and PTA were used in 5 patients and 22 patients, respectively. Fourteen patients had stenosis and 13 patients had occlusion. The total procedural success rate was $81 \%, 86 \%$ in stenosis and $77 \%$ in occlusion. There was no procedure-related complication. The primary patency for PTA at 6 and 12 months were $40 \%$ and $10 \%$, respectively. For stent implantation, primary patency rate at 6 and 12 months was $70 \%$ and $30 \%$, respectively.

Conclusion: Endovascular interventions for central venous stenosis and occlusion are safe, with low rates of technical failure and they can be first-line treatment for central venous stenosis or occlusion in hemodialysis patients.
\end{abstract}

Key words: central venous stenosis, central venous occlusion, endovascular intervention, percutaneous transluminal angioplasty, stent implantation

Acta Angiol 202I; 27, I: |7-2|

\section{Introduction}

Central (superior vena cava, brachiocephalic, or subclavian) vein stenosis and occlusion are an important complication in hemodialysis patients. Central venous stenosis or occlusion usually occurs as a complication of central venous catheterization and significantly complicates dialysis. Clinically, central venous stenosis or occlusion manifests as ipsilateral arm or neck swelling and failure of hemodialysis access.

Surgical and endovascular treatments are available for the treatment of central venous stenosis or occlusion. Surgical treatment is often difficult and may not always be successful. Surgical approaches require general anesthesia and have high surgical morbidity in patients with end-stage renal disease. However, percutaneous treatment for central venous stenosis is feasible and efficient. Surowiec et al. [I] showed that transvenous angioplasty was helpful for hemodialysis patients with central venous stenosis and helped maintain functional access in the affected limb. Endovascular treatment options include percutaneous transluminal angioplasty (PTA) or stent implantation. The optimal endovascular treatment remains unclear; the superiority of stent placement compared with PTA has not been fully established [1-3]. The National Kidney Foundation Disease Outcomes Quality Initiative guidelines have recommended angioplasty as initial treatment; stenting is indicated in case of central vein stenosis recurring within 3 months $[4,5]$. 

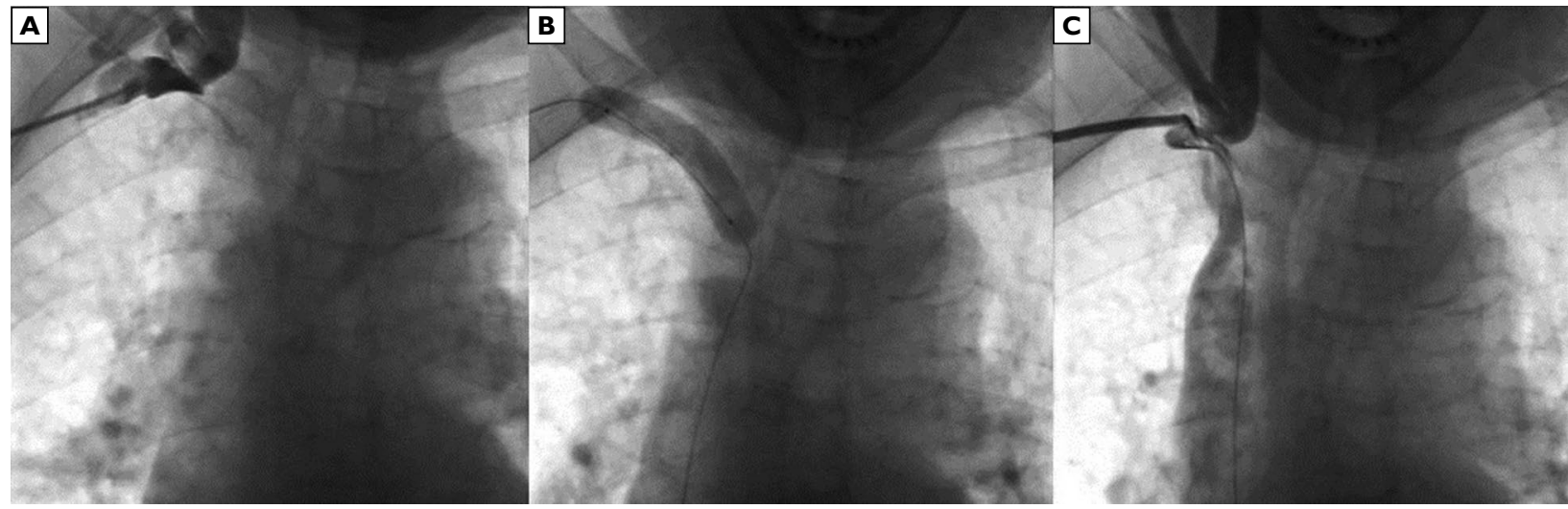

Figure I. A 58-year-old patient with a history of 10-year hemodialysis. A - initial diagnostic venogram showed complete occlusion of right subclavian vein; B - the lesion was crossed anterogradely using guide wire, and PTA was performed; C - post PTA venogram showed normal filling of right subclavian vein. During follow-up, the patient had not any recurrence at 12 months

The aim of the study was to determine 6-month and 12 -month primary patency of percutaneous balloon angioplasty and stent implantation in hemodialysis patients who had central venous stenosis or occlusion.

\section{Material and methods}

This study was a retrospective, single-center study based on collected data of endovascular interventions which were PTA or stent implantation for central venous stenosis or occlusion. Data were collected from the interventional radiology department database from January 2013 to January 2018. The study was approved by the ethics committee. Written informed consent was obtained from all the patients.

PTA or stent implantation was performed in endstage renal failure patients on hemodialysis who had clinical signs of central venous stenosis or occlusion. All patients had a history of central hemodialysis catheter placement. Clinical signs in these patients were prolonged post-hemodialysis hemorrhage, increased venous pressure, ipsilateral arm or neck swelling and decreased blood flow during dialysis sessions. Patients who had previous PTA or stent implantation and previous surgical treatment for the same lesion were excluded from the study. In all patients, the location and length of the stenosis or occlusion were evaluated by diagnostic venography before the intervention.

Endovascular interventions were started with antegrade puncture with an I8-G needle into the proximal stenotic/occlusive vein and a 7-French vascular sheath was inserted via this vein. Antegrade venous access through the common femoral vein was added to the procedure in difficult cases. A total dose of $3000 \mathrm{IU}$ heparin was injected via the vascular sheath to prevent thrombus formation. The stenotic site was traversed using a 0.035 -inch hydrophilic guidewire (Terumo, NJ, USA). For some hard lesions, different guidewires, such as stiff guidewire or 0.018 -inch guidewire or microcatheter, were also used for passing the stenosis/ locclusion. PTA or stent implantation was performed after traversing the lesion. Depending on the size of the vessels, PTAs were I $\mathrm{mm}$ larger (ranging from 10 to $14 \mathrm{~mm}$ ) than the normal size of the venous segment, and the length of the balloons ranged from 2 to $8 \mathrm{~cm}$ (Fig. I). Stenting was performed if a residual stenosis of greater than $50 \%$ was present after PTA. Only bare selfexpanding metal stents (Wallstent, Boston Scientific, Natick, MA, USA) were used and the diameter of the stent was chosen nearly the same as the adjacent normal vein (Fig. 2). Stent diameters ranged from 10 to $14 \mathrm{~mm}$, with length ranging from 4 to $6 \mathrm{~cm}$. Post-dilatation with a balloon often was performed after stent deployment to improve stent expansion. A final angiogram was performed to evaluate residual stenosis and technical failure was defined as inability to cross the stenotic or occlusive segment and residual stenosis $>30 \%$.

The primary patency was defined as patency in the patent central vein without recurrent stenosis or occlusion. Assisted primary or secondary patency was defined as a patent central vein that underwent further intervention to improve patency.

\section{Statistical analysis}

Statistical analyses were performed using Statistical Package for the Social Sciences (SPSS) 17.0 statistical software for Windows (SPSS Inc., Chicago, IL, USA). The chi-square test and Kaplan-Meier analysis were used to calculate primary patency of the PTA and stent implantation in the groups (Fig. 3). 


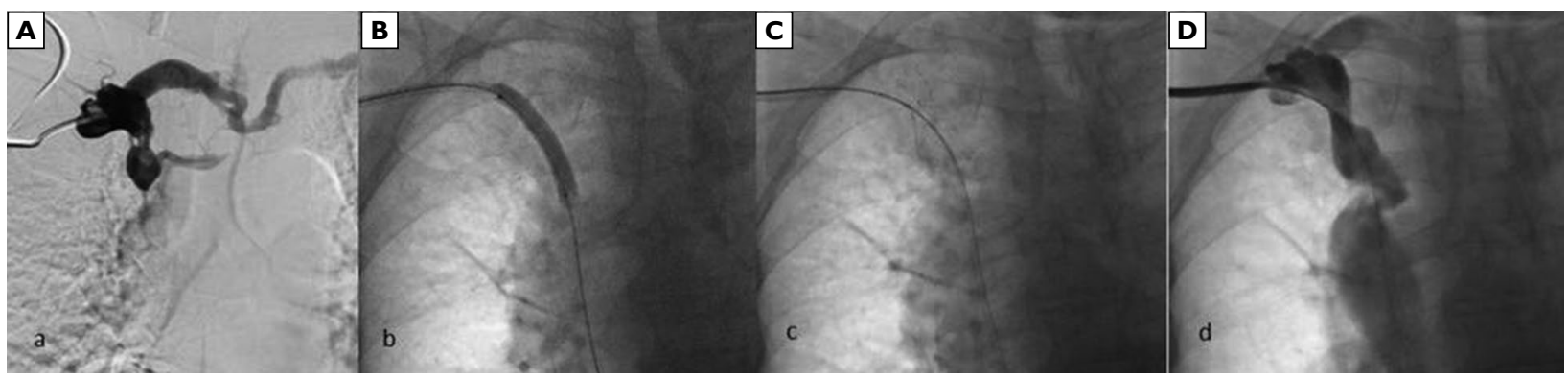

Figure 2. A 66-year-old patient with a history of 8-year hemodialysis. A — initial diagnostic venogram showed serious stenosis of superior vena cava. B, C - predilatation and stent implantation was performed. D - post procedure venogram showed normal filling of superior vena cava. At follow-up, restenosis was seen at 8 months

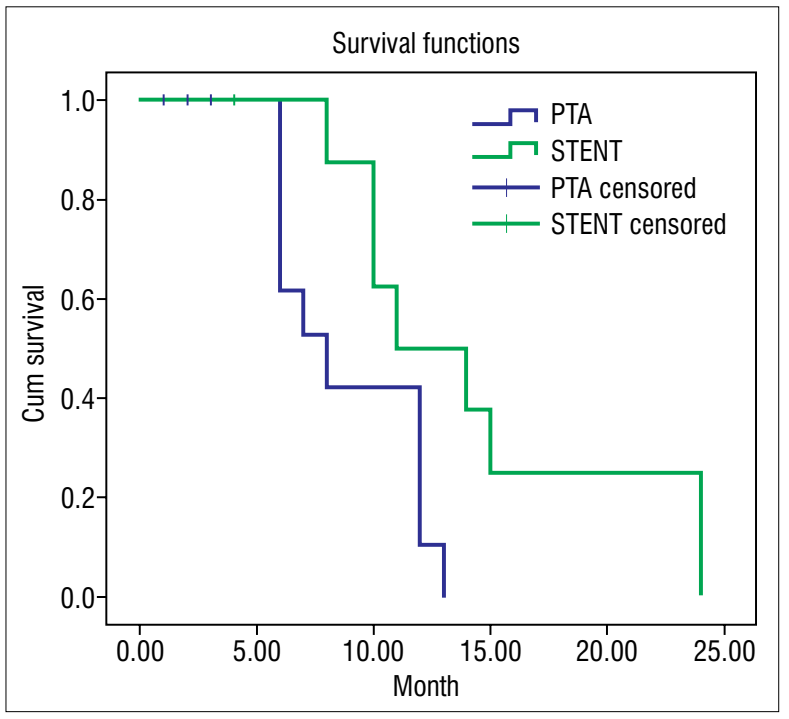

Figure 3. Kaplan-Meier graphs: a comparison of 12-month primary patency rates between PTA and stent implantation

\section{Results}

Endovascular procedures were performed in 27 hemodialysis patients with central venous stenosis or occlusion. There were $14(52 \%)$ males and $13(48 \%)$ females. The mean age of the patients was $62.2 \pm 14$ years (range, 35-87 years). Stent implantation was used for 5 patients and PTA for 22 patients. Before endovascular treatment, 14 patients had stenosis and 13 had occlusion. Only PTA was performed in $86 \%$ of 14 patients with venous stenosis and $77 \%$ of $I 3$ patients with occlusion, while other patients underwent stent angioplasty. The sites of central vein stenosis or occlusion were the superior vena cava in II cases, brachiocephalic vein in 15 cases and the subclavian vein in I case. Demographic characteristics of the patients are summarized in Table I. The overall procedural success
Table I. Demographic data and procedural details of patients

\begin{tabular}{|c|c|}
\hline Mean age of patients & $66 \pm 14$ years \\
\hline Sex (female) & $13(48 \%)$ \\
\hline Mean duration of hemodialysis & 8 years \\
\hline \multicolumn{2}{|l|}{ Type of lesions } \\
\hline Stenosis & $14(52 \%)$ \\
\hline Occlusion & $13(48 \%)$ \\
\hline \multicolumn{2}{|l|}{ Site of lesions } \\
\hline Superior vena cava & II (40\%) \\
\hline Brachiocephalic vein & 15 (55\%) \\
\hline Subclavian vein & I (3\%) \\
\hline \multicolumn{2}{|l|}{ Mean length of the lesions } \\
\hline Stenosis & $36.2 \pm 6.2 \mathrm{~mm}$ \\
\hline Occlusion & $28.5 \pm 4.3 \mathrm{~mm}$ \\
\hline \multicolumn{2}{|l|}{ Endovascular therapy } \\
\hline Percutaneous balloon angioplasty & $22(8 \mid \%)$ \\
\hline Stent implantation & $5(19 \%)$ \\
\hline \multicolumn{2}{|l|}{ Size of balloons used } \\
\hline Superior vena cava ( 8 patient) & $\begin{array}{l}\mathrm{I} 2 * 40 \mathrm{~mm}(\mathrm{x} 4) \\
\mathrm{I} 2 * 60 \mathrm{~mm}(\times 2) \\
\mathrm{I} 4 * 40 \mathrm{~mm}(\times 2)\end{array}$ \\
\hline Brachiocephalic vein( 13 patient $)$ & $\begin{array}{l}12 * 40 \mathrm{~mm}(\times 6) \\
10 * 40 \mathrm{~mm}(\times 3) \\
10 * 60 \mathrm{~mm}(\times 4)\end{array}$ \\
\hline Subclavian vein (I patient) & $8 * 40 \mathrm{~mm}(\mathrm{x} \mathrm{I})$ \\
\hline \multicolumn{2}{|l|}{ Size of stents used } \\
\hline Superior vena cava ( 3 patient) & $\begin{array}{l}\mathrm{I} 2 * 40 \mathrm{~mm}(\times 2) \\
\mathrm{I} 4 * 40 \mathrm{~mm}(\times \mathrm{I})\end{array}$ \\
\hline Brachiocephalic vein (2 patient) & $12 * 40 \mathrm{~mm}(\times 2)$ \\
\hline
\end{tabular}

Balloons and stents are expressed as diameter * length in $\mathrm{mm}$

rate for PTA and stent implantation was $83 \%$; $100 \%$ in stenosis and $70 \%$ and $100 \%$ in occlusion. After balloon angioplasty, residual stenosis greater than $30 \%$ 
was detected in the control angiograms in 2 patients who had stenosis and 3 patients who had occlusion, which were considered as technical failure. The total success rate for all procedures was $81 \%$. There were no procedure-related complications and all patients underwent hemodialysis after the initial procedure.

The primary patency for PTA at 6 and 12 months was $40 \%$ and $10 \%$, respectively. For stent implantation, primary patency at 6 and 12 months was $70 \%$ and $30 \%$, respectively. The mean reintervention time was 7 months for PTA and II months for stent implantation. The mean reintervention time was II months in patients with stenosis and 6 months in patients with occlusion. There was no statistically significant difference between balloon angioplasty and stent implantation groups according to the chi-square test and Kaplan-Meier analysis ( $p>0.05)$.

In the PTA group, $20(91 \%)$ initial PTAs required secondary intervention during the 12-month follow-up period due to loss of primary patency. Secondary PTA was used in 18 patients and 2 patients underwent stent implantation. In the stent implantation group, 3 (70\%) patients required secondary intervention and PTA was applied to these patients. Total reinterventions in both groups were required 23 (85\%) patients during the I 2-month follow-up period, but only 8 patients were followed up 6 month after second intervention and secondary patency was seen $75 \%$ at 6 months.

\section{Discussion}

The number of patients with chronic renal failure requiring hemodialysis is increasing and a smooth vascular structure is required for successful dialysis. Vascular complications are one of the main causes associated with an increase in morbidity and mortality in hemodialysis patients. Central venous stenosis and occlusion are a major concern in patients receiving prolonged hemodialysis. There are various surgical approaches to management of central venous stenosis or occlusion such as interposition grafting to the internal jugular vein, and direct patch angioplasty of axillo-subclavian stenosis which include jugular venous turndown. The results of surgical reconstruction of the central veins are better than those of endovascular therapy with primary patency rates of $80 \%$ to $90 \%$ at one year [6]. However, these surgical approaches are difficult to perform in hemodialysis patients with numerous comorbidities. Central veins are generally obscured by the bony skeleton and these major surgeries often require clavicular division or sternotomy along with general anesthesia. Endovascular treatments are less invasive and therefore preferred over surgical treatment in many centers. Exceptionally, subclavian vein stenoses adjacent to the costoclavicular junction respond poorly to treatment with endovascular therapy because of extrinsic compression at the thoracic outlet. The optimal treatment of access-related venous stenosis at this location must include a transaxillary first rib resection and mobilizing the subclavian vein to the jugular confluence [7]. Endovascular therapy is not usable only for benign central venous stenosis and occlusion but also for malignant processes. Also, surgical resection and reconstruction of the superior vena cava in selected patients with malignant mediastinal tumors may be very beneficial [8].

One of the negative consequences of endovascular treatment is acceleration of occlusion formation. Recurrent lesions after angioplasty have more aggressive neointimal hyperplasia than the primary lesion because the mechanism of angioplasty involves cracking and fissuring of the vessel intima which can increase neointimal hyperplasia [9]. The endovascular therapy options include balloon angioplasty and stent implantation. Although, transluminal angioplasty is the preferred treatment for central venous stenosis or occlusion [4], optimal endovascular management strategy is unclear in many centers.

In this study, the total procedural success rate was found to be $81 \%$; it ranges between $70 \%$ and $90 \%$ in the literature [1, 2, 10-12]. The primary patency for PTA at 6 and 12 months was $40 \%$ and $10 \%$, respectively. For stent implantation, primary patency at 6 and 12 months was $70 \%$ and $30 \%$, respectively. Previous studies showed that primary patency rates for PTA ranged between $23 \%$ and $55 \%$ at 6 months and $12 \%$ and $50 \%$ at 12 months and primary patency rates for stent implantation ranged from $42 \%$ to $89 \%$ at 6 months, and from $14 \%$ to $73 \%$ at 12 months [I-3, II, I3-16]. Our results were correlated with these studies. In our study, secondary or assisted primary patency rate was $75 \%$ during the 6-month follow-up.

As we know, stent implantation has some late complications such as stent fracture and migration of the distal stent fragment to the inferior vena cava or stent compression distortion. In our cases, there were no stent-related late complications and all patients underwent hemodialysis after the initial procedure. In our study cohort, only bare self-expanding metal stents were used. The bare self-expanding metal stent (Wallstent) excels as a conduit, rarely fracturing or failing primarily through the main body of the stent. The stent maintains strength via both radial force and compression resistance. Although the bare self-expanding metal stent has proven advantageous, there remain two critical shortcomings. First, the edges of the stent are weaker than the main body, making it prone to collapse. And he second is the lack of deployment accuracy and precision, making it prone to stent forshortening. In our 
cases, there were no collapse and significant shortening of stent after procedure. New generation nitinol venous stents design is advantageous for improved precision and accuracy during deployment without significant stent forshortening. Studies comparing PTA and stenting in central venous lesions reported that the patency rates in central lesions were equal at 12 months [2, 3]. Restenosis is common after PTA or stent implantation, therefore, cost effectiveness is now being questioned in many centers. Stent implantation in our country is 3-4 times more expensive than PTA. So, we prefer PTA firstly in hemodialysis patients with initial stenosis and occlusion. However, in patients with residual stenosis greater than $50 \%$ after PTA, stent implantation was performed.

Mid-term primary patency rate of endovascular intervention and surgical treatment are similar in central stenosis or occlusion, with a significant incidence of secondary interventions [17]. It would be beneficial to choose endovascular intervention as a first-line treatment because of its less invasiveness and cost-effectiveness.

Our study has certain limitations. First, our sample size was small. It was necessary to collect data over a long time period to have a sufficient number of cases for statistical power. Additional limitations were retrospective nature of the study, availability of only limited width bare self-expanding metal stents and absence of surgically treated cases.

\section{Conclusion}

Although long-term patency rates of endovascular interventions in central venous stenosis and occlusion in hemodialysis patients are not satisfactory, because of less invasive nature and high technical success rate, it can be chosen as a first-line treatment method.

\section{Conflict of interest}

None.

\section{References:}

I. Surowiec SM, Fegley AJ, Tanski WJ, et al. Endovascular management of central venous stenoses in the hemodialysis patient: results of percutaneous therapy. Vasc Endovascular Surg. 2004; 38(4): 349-354, doi: 10.1177/153857440403800407, indexed in Pubmed: 15306953.

2. Bakken $A M$, Protack CD, Saad WE, et al. Long-term outcomes of primary angioplasty and primary stenting of central venous stenosis in hemodialysis patients. J Vasc Surg. 2007; 45(4): 776-783, doi: 10.1016/j.jvs.2006.12.046, indexed in Pubmed: 17398386.

3. Quinn SF, Schuman ES, Demlow TA, et al. Percutaneous transluminal angioplasty versus endovascular stent placement in the treatment of venous stenoses in patients undergoing hemo- dialysis: intermediate results. J Vasc Interv Radiol. 1995; 6(6): 85 I-855, doi: 10.1016/s 105I-0443(95)7I 200-3, indexed in Pubmed: 8850659.

4. III. NKF-K/DOQI Clinical Practice Guidelines for Vascular Access: Update 2000. American Journal of Kidney Diseases. 200I; 37(I): SI37-SI8I, doi: 10.1016/s0272-6386(0I)70007-8.

5. Vascular Access Work Group, Vascular Access 2006 Work Group. Clinical practice guidelines for vascular access. Am J Kidney Dis. 2006; 48 Suppl I: S176-S247, doi: 10.1053/j. ajkd.2006.04.029, indexed in Pubmed: I68I3989.

6. Mickley V. Central vein obstruction in vascular access. Eur J Vasc Endovasc Surg. 2006; 32(4): 439-444, doi: 10.1016/j. ejvs.2006.04.0II, indexed in Pubmed: 16765068.

7. Wooster M, Fernandez B, Summers KL, et al. Surgical and endovascular central venous reconstruction combined with thoracic outlet decompression in highly symptomatic patients. J Vasc Surg Venous Lymphat Disord. 2019; 7(I): 106-I I2.e3, doi: 10.1016/j. jvsv.2018.07.019, indexed in Pubmed: 30442583.

8. Kaba E, Özkan B, Özyurtkan MO, et al. Superior vena cava resection and reconstruction in mediastinal tumors and benign diseases. Turk Gogus Kalp Damar Cerrahisi Derg. 2018; 26(I): 99-107, doi: 10.5606/tgkdc.dergisi.2018.14292, indexed in Pubmed: 32082718.

9. Chang CJ, Ko PJ, Hsu LA, et al. Highly increased cell proliferation activity in the restenotic hemodialysis vascular access after percutaneous transluminal angioplasty: implication in prevention of restenosis. Am J Kidney Dis. 2004; 43(I): 74-84, doi: 10. 1053/j. ajkd.2003.09.015, indexed in Pubmed: 147| 2430.

10. Yadav MK, Sharma M, Lal A, et al. Endovascular treatment of central venous obstruction as a complication of prolonged hemodialysis - Preliminary experience in a tertiary care center. Indian J Radiol Imaging. 2015; 25(4): 368-374, doi: 10.4103/097I3026.169463, indexed in Pubmed: 26752817.

II. Aj A, Razak Uk A, R P, et al. Percutaneous intervention for symptomatic central vein stenosis in patients with upper limb arteriovenous dialysis access. Indian Heart J. 2018; 70(5): 690-698, doi: 10.1016/j.ihj.2018.01.013, indexed in Pubmed: 30392508.

12. Dammers R, de Haan MW, Planken NR, et al. Central vein obstruction in hemodialysis patients: results of radiological and surgical intervention. Eur J Vasc Endovasc Surg. 2003; 26(3): 317-32I, doi: 10.1053/ejvs.2002.1943, indexed in Pubmed: 14509897.

13. Nael K, Kee ST, Solomon H, et al. Endovascular management of central thoracic veno-occlusive diseases in hemodialysis patients: a single institutional experience in 69 consecutive patients. J Vasc Interv Radiol. 2009; 20(I): 46-5I, doi: 10.1016/j. jvir.2008.09.020, indexed in Pubmed: 19019699.

14. Haage P, Vorwerk D, Piroth W, et al. Treatment of hemodialysis-related central venous stenosis or occlusion: results of primary Wallstent placement and follow-up in 50 patients. Radiology. 1999; 212(1): 175-180, doi: 10.1148/radiology.212.1.r99j|21 I75, indexed in Pubmed: 10405739.

15. Chen CY, Liang HL, Pan HB, et al. Metallic stenting for treatment of central venous obstruction in hemodialysis patients. J Chin Med Assoc. 2003; 66(3): 166-172, indexed in Pubmed: 12779037.

16. Vorwerk D, Guenther RW, Mann H, et al. Venous stenosis and occlusion in hemodialysis shunts: follow-up results of stent placement in 65 patients. Radiology. 1995; 195(I): 140-146, doi: 10.1 I 48/radiology. 195.I.7892456, indexed in Pubmed: 7892456.

17. Sfyroeras GS, Antonopoulos CN, Mantas G, et al. A Review of Open and Endovascular Treatment of Superior Vena Cava Syndrome of Benign Aetiology. Eur J Vasc Endovasc Surg. 2017; 53(2): 238-254, doi: 10.1016/j.ejvs.2016.11.013, indexed in Pubmed: 28007450 . 